\title{
Using Temporal Information for Improving Articulatory-Acoustic Feature Classification
}

\author{
Barbara Schuppler, Joost van Doremalen, Odette Scharenborg, Bert Cranen, Lou Boves \\ Centre for Language and Speech Technology, Radboud University Nijmegen, The Netherlands \\ \{B.Schuppler, J.vanDoremalen, O.Scharenborg, B.Cranen, L.Boves\}@let.ru.nl
}

\begin{abstract}
This paper combines acoustic features with a high temporal and a high frequency resolution to reliably classify articulatory events of short duration, such as bursts in plosives. SVM classification experiments on TIMIT and SVArticulatory showed that articulatory-acoustic features (AFs) based on a combination of MFCCs derived from a long window of $25 \mathrm{~ms}$ and a short window of $5 \mathrm{~ms}$ that are both shifted with $2.5 \mathrm{~ms}$ steps (Both) outperform standard MFCCs derived with a window of 25 ms and a shift of $10 \mathrm{~ms}$ (Baseline). Finally, comparison of the TIMIT and SVArticulatory results showed that for classifiers trained on data that allows for asynchronously changing AFs (SVArticulatory) the improvement from Baseline to Both is larger than for classifiers trained on data where AFs change simultaneously with the phone boundaries (TIMIT).
\end{abstract}

\section{INTRODUCTION}

Most automatic speech recognition (ASR) systems are based on the principle that words are composed of a sequence of phones, also referred to as the 'beads on a string' model of speech [1]. This model works reasonably well for carefully produced speech. However, ASR performance drops tremendously for spontaneous speech, mainly due to the high pronunciation variability [2]. Phone-based modeling of pronunciation variation has its limitations, because it is not able to capture the overlapping, asynchronous gestures of the articulators, e.g., [3]. Therefore, there has been an increased interest in articulatory-acoustic features (AFs), which are the acoustic correlates of articulatory events. With this type of features speech can be represented in a way that does not impose a sequence of discrete segments. An estimate of the degree of asynchrony in AF changes in speech is given in [4] in terms of AF combinations. AF representations derived from the canonical phonemic transcriptions resulted in 62 AF combinations. When the AFs were allowed to change asynchronously, this number increased to 351 .

AF classifiers have been used to improve speech recognition performance in adverse conditions [5], [6], to build language independent phone recognizers [7], and to improve computational models of human word recognition [8]. Furthermore, AF-based descriptions of the speech material are now being used to investigate pronunciation errors by learners of a second language [10] and for the automatic analysis of fine-phonetic detail [11]. For these latter two applications, an accurate and reliable classification is crucial.

Unfortunately, the evaluation of the performance of AF classifiers suffers from the absence of large corpora that provide reliable labeling of AF values. As a consequence, training and testing of AF classifiers is generally done on the basis of data that is labeled on the phoneme level after which all phonemes are replaced by their (canonical) AF values. Thus, these phonologically inspired AF values change synchronously at phone boundaries. Obviously, it is unclear to what extent the classifiers trained with AFs obtained in this fashion can be assumed to yield classification results that truly reflect articulatory gestures.

The aim of the present study is two-fold. The first aim is to build an AF classifier that can be used for reliable and accurate detection of slight pronunciation errors and the automatic analysis of fine-phonetic detail. One error second language learners often make is the confusion of fricatives and plosives [12]. Moreover, plosives show tremendous articulatory variation in casual speech [11]. Therefore, in this paper, we focus on improving the automatic classification of the manner of articulation (see Section I.A.). The second aim is to investigate the effect of the AF labeling of the training and test material on performance estimates (see Section I.B).

\section{A. Improving manner classification through capturing tem- poral information in the acoustic features}

Previous research has attempted to improve AF classifiers from several directions. First, different statistical classifiers have been tested and their (frame-based) classification accuracies have been compared [5], [13], [14]. Second, different methods to parameterize the acoustic waveforms have been evaluated for the task of AF classification [5], [15]. A third type of approach tries to exploit the long span contextual influence among phonetic units by explicit modeling of the dynamic temporal constraints induced by the physical properties of the articulators (e.g. [27] and the references therein).

Regardless of the AF classification system used, the underlying acoustic representation are mostly MFCCs and hardly anyone has moved away from using a window length of $25 \mathrm{~ms}$ and a window shift of $10 \mathrm{~ms}^{1}$, probably because these settings are known to be close to optimal for ASR purposes. With this window length and shift reasonably good results can be obtained for more or less stationary sounds, for whose correct classification a high resolution in the frequency domain is more important than a high temporal resolution. For instance, around $90 \%$ of the vowels were classified correctly in a classification task [13] on TIMIT [18]. However, many articulatory events are not stationary or are much shorter than $25 \mathrm{~ms}$ and can therefore not be properly captured. This is especially the case for plosives. For comparison, the same study [13]

${ }^{1}$ To our knowledge, only two studies used a different window length and shift: [16] used a $16 \mathrm{~ms}$ window and a $8 \mathrm{~ms}$ shift, while [17] used a $25 \mathrm{~ms}$ window shifted with $2.5 \mathrm{~ms}$. 
showed that only $80 \%$ of the plosives were correctly classified. In the TIMIT database, which is often used for investigation of AFs, $10 \%$ of the segments are shorter than $25 \mathrm{~ms}$.

In order to obtain both a high time and frequency resolution, we propose an acoustic feature vector with MFCCs derived from a window of $25 \mathrm{~ms}$ and from a short window of $5 \mathrm{~ms}$ that are both shifted with $2.5 \mathrm{~ms}$ steps. We will investigate the usefulness of such extended acoustic features by training an AF classifier based on a support vector machine (SVM) and subsequently test it on TIMIT. The reason for using an SVM is that this type of classifiers provides good generalization given a small amount of high-dimensional data, and that SVMs have shown good AF classification results (e.g., [13]). The classifier trained with the new acoustic feature vectors based on a combination of short and long windows will be compared to a baseline classifier with 'standard' MFCCs in order to investigate whether these acoustic features achieve a better frame classification accuracy.

\section{B. Dealing with inaccurate material}

Ideally, AF classifiers should be trained and tested on observed articulatory trajectories or on speech corpora that are manually transcribed on the articulatory level. However, these data are not available in sufficient quantity, and the creation of these data is extremely time-consuming. For instance, [20] reported that transcriptions of utterances on the AF level take 1000 times real-time for SVitchboard [21] (a selection of the Switchboard corpus that can be considered a small vocabulary data set). The conventional 'solution' to obtain enough training and testing material is to use a canonical mapping from phonetic alignments to AF values. Thus, the phoneme /t/ would map to the AF values 'voiceless', 'alveolar', and a sequence of 'closure' and 'burst+release'. However, for Dutch, it has been shown that only $11.5 \%$ of word-final $/ \mathrm{t} / \mathrm{s}$ are realized according to this canonical feature representation [11]. Similar pronunciation variation can be expected for other sounds and in other languages. Thus, the mapping from phonemic labels to AF labels results in classifiers that are trained and tested on stretches of speech that may not contain the assigned feature value at all. This raises doubts about the validity of the data the classifiers are trained and evaluated on.

In the absence of a sufficient amount of AF labeled data, we followed the standard procedure: the reference frame labels are derived by replacing the frame-level phonemic TIMIT labels by the canonical AF values. This thus implies that if one wants to evaluate the degree of success with which a classifier is able to correctly classify the acoustic correlates of underlying articulatory gestures, while in actual practice the phone-based canonical labels are used as the reference, asynchronously changing AFs may be erroneously marked as errors. The impact on apparent frame accuracy due to the lack of a transcription that accounts for asynchronously changing AFs is illustrated by [22]. They showed that if a feature is allowed to change within a range of \pm 2 frames from the phone boundary, the measure "all frames correct" increases significantly by $9 \%$ to $63 \%$. Therefore, the number of such virtual errors occurring at phone boundaries creates a substantial (and for diagnostic purposes, misleading) decrease in the frame ac- curacy when not allowing asynchronously changing AFs. Note that the lack of a transcription of the speech signal that accounts for asynchronously changing AFs also means that it is impossible to achieve $100 \%$ correct classification on the given task and that the 'upper-bound' of the classification accuracy is unknown. In order to investigate the effect of the synchronously changing AF values around phone boundaries on the errors the AF classification systems make, we carry out an analysis of the classification results around the phone boundaries.

Finally, the fact that an AF classifier is often trained on stretches of speech that may or may not contain the assigned feature value can result in an AF classifier that suggests a good performance in detecting plosives, even if not all plosives are produced in their canonical form as a closure and a release. Since our goal is to build classifiers that are able to detect slight pronunciation errors and fine-phonetic detail, it is important that the classifier can distinguish different plosive realizations. Therefore, in addition to evaluating the classifier on the TIMIT data, we also test the new acoustic features on those SVitchboard utterances that have been transcribed at the AF value level [20]. In this paper we refer to the latter dataset as SVArticulatory. Comparing the new classifier with the baseline system on both data sets will show whether the new classifier is better able to describe the speech signal at the articulatory feature level.

TABLE 1

\begin{aligned} & MAPPING OF TIMIT PHONE SYMBOLS TO THE MANNER AF VALUES. \\ & \hline Phone Manner AF value \\ & \hline sil, pau silence \\ & l, el, r liquid \\ & $\mathrm{w}, \mathrm{y}$ glide \\ & $\mathrm{em}, \mathrm{en}, \mathrm{eng}, \mathrm{m}, \mathrm{n}, \mathrm{ng}, \mathrm{nx}$ nasal \\ & $\mathrm{dh}, \mathrm{f}, \mathrm{hh}, \mathrm{s} \mathrm{sh}, \mathrm{th}, \mathrm{v}, \mathrm{z}, \mathrm{zh}, \mathrm{hv}$ fricative \\ & $\mathrm{b}, \mathrm{d}, \mathrm{g}, \mathrm{p}, \mathrm{t}, \mathrm{k}, \mathrm{q}$ burst+release \\ & bcl, dcl, gcl, pcl, tcl kcl closure \\ & $\mathrm{dx}, \mathrm{epi}$, all vowels NIL \\ & \hline\end{aligned}

\section{METHOD}

\section{A. Articulatory feature values}

In the past, different methods have been proposed to characterize manner of articulation. For example, plosives can either be mapped as a whole on one AF value 'plosive' [13], [22], [23] or be split up into two parts 'closure' and 'release', where the release can be modeled together with 'fricative' [5], [24] or separate from the fricatives as a 'burst+release' [14], [16]. The latter is also the way we deal with plosives, for two reasons. First, modeling plosives as one unit violates the assumption of SVMs that the sequence of frames assigned to a sound can be considered as drawn from one population, which is definitely not the case for plosives that consist of a sequence of 'closure' and 'release'. Secondly, we aim at using AF classifiers to analyze how the sounds were actually produced, for instance whether a plosive was realized as a closure followed by friction or as a closure plus burst and friction. 
Therefore we train separate classifiers for 'fricative' and 'burst+release'. We excluded all 'vowel' material, because it is possible that certain AF values overlap in time, for instance in the case of nasalized vowels. However, when including both 'vowel' and 'nasal' as an AF value, these AF values cannot co-occur in a frame, as the classifier has to make a choice between the two. A full overview of the manner AF values is given in Table 1.

\section{B. Speech material}

\section{1) TIMIT}

The speech material used in this study is taken from TIMIT, which contains hand-labeled and hand-segmented phonetically balanced sentences read by 630 speakers (of which $70 \%$ were male) from eight major dialects of American English. We followed TIMITs training and testing division, in which no sentence or speaker appears in both the training and test set. The training set consists of 3,696 utterances.

To train the SVM classifiers, a smaller training set of $25,21010 \mathrm{~ms}$ frames was created by randomly selecting frames from the full training set with the same prior distribution of the AF value classes as in the full training set. Note: since the window shift is $2.5 \mathrm{~ms}$ in the case of the new acoustic features, the original 25,210 training frames were split into $100,8422.5 \mathrm{~ms}$ frames to train the new classifiers.

In the first experiment (Section III.A.), the classifiers were tested on the TIMIT test set consisting of 1,344 utterances; i.e., 236,984 10ms frames and 943,604 2.5ms frames.

The TIMIT database is labeled using 59 Arpabet symbols, which have been relabeled in terms of AF values according to Table 1 . Therefore, the AF values change simultaneously with the original phone boundaries. Note that it is possible that segments that have been annotated as 'burst+release' in the TIMIT material indeed contain a burst; however, it is also possible that the burst is actually missing, just leaving frication.

\section{2) SVArticulatory}

For the second experiment, we used a data set that consisted of 78 utterances (a total of 119s of speech, excluding initial and final silences) drawn from SVitchboard, a smallvocabulary subset of spontaneous telephone speech from Switchboard [19]. This subset is converted into a set of 13,295 $10 \mathrm{~ms}$ frames, and a set of 53,115 $2.5 \mathrm{~ms}$ frames. SVArticulatory was manually transcribed on the AF level for the 2006 JHU Summer Workshop [20]. The original set of AFs did, however, not match our set of AFs. Therefore, manual adaptations were made for which the starting point was the transcriptions from the tier on which the feature set ' $\mathrm{Dg} 1$ ' (Degree of forward constriction) was annotated. These transcriptions were modified to our feature set. Table 2 shows the mapping between these two feature sets. When modifying the annotations, the original boundaries were maintained, but the labels were changed according to Table 2; e.g., the original label 'fricative' was changed to 'burst+release' in those plosives where a burst was present. Releases without burst maintained the label 'fricative'. New boundaries were placed when one feature in the ' $\mathrm{Dg} 1$ ' set is transcribed as two features in our set; e.g., an 'approximant' in the original set occasionally was replaced by a 'liquid' followed by a 'glide' in our set. New boundaries were placed to separate background speakers from silence. The boundaries of the 'Nasality' tier of the original transcription were used to annotate the nasal consonants. Previous labeling mistakes that occurred in two utterances were corrected. Furthermore, frames labeled as flap or containing background speech have been removed.

TABLE 2

PHONE-TO-AF MAPPING FOR THE ORIGINAL 'DG1'(DEGREE OF FORWARD CONSTRICTION) AND OUR AF SET.

\begin{tabular}{rll}
\hline Phone & Dg1 & Our AF set \\
\hline l, el & closure & liquid \\
er, r & approximant & liquid \\
$\mathrm{w}, \mathrm{y}$ & approximant & glide \\
$\mathrm{em}, \mathrm{en}, \mathrm{eng}, \mathrm{m}, \mathrm{n}, \mathrm{ng}, \mathrm{nx}$ & closure & nasal \\
$\mathrm{dh}, \mathrm{f}, \mathrm{hh}, \mathrm{s} \mathrm{sh}, \mathrm{th}, \mathrm{v}, \mathrm{z}, \mathrm{zh}, \mathrm{hv}$ & fricative & fricative \\
$\mathrm{b}, \mathrm{d}, \mathrm{g}, \mathrm{p}, \mathrm{t}, \mathrm{k}, \mathrm{q}$ & fricative & burst+release \\
& & or fricative \\
& closure \\
$\mathrm{bcl}, \mathrm{dcl}, \mathrm{gcl}, \mathrm{pcl}, \mathrm{tcl} \mathrm{kcl}$ & closure & silence \\
silence & silence &
\end{tabular}

TABLE 3

THE $\%$ SV AND VALUES FOR C AND $\gamma$ FOR DIFFERENT ACOUSTIC FEATURES.

\begin{tabular}{lccc}
\hline & \%SV & $\boldsymbol{c}$ & $\boldsymbol{\gamma}$ \\
\hline Baseline & 55.0 & 0.4 & 2 \\
Short & 46.1 & 0.8 & 1 \\
Long & 31.1 & 0.5 & 4 \\
Both & 41.8 & 0.3 & 2 \\
\hline
\end{tabular}

\section{Support Vector Machines}

The AF classifiers built in this study are SVMs [25]. In our experiments, we used the LIBSVM package [26], which achieves multi-class classification by error correcting codes. The RBF kernel was used for the experiments reported in this paper.

For the AF classifiers, trained using four sets of acoustic features (cf. Section III.A), the number of support vectors (SVs) is listed in Table 3 as a percentage of the amount of training data $(\% \mathrm{SV})$. The percentage of SVs indicates the task complexity: more SVs suggest either more complex decision boundaries or more overlapping data. Table 3 also lists the $\gamma$ and $c$ parameters in the SVMs, estimated on an independent development set of 2,000 frames. A large $\gamma$ implies narrower RBFs. If $c$ is large, the more complex decision boundaries are constructed to fit the training data, but this may result in poor generalization.

Table 3 shows that the $\% \mathrm{SV}$ is lowest for Long, while the value for $\gamma$ is highest. This indicates that the width of the RBFs is reasonably small, which suggests that the clusters in the Long model are more localized, with little overlap between the AF values, resulting in a fair generalization. The $c$ values of the four models do not differ much. The $\% \mathrm{SV}$ is highest for Baseline, while the value for $\gamma$ is relatively low, indicating that the width of the RBFs is relatively large which results in less localized AF clusters and less generalization than Long and 
Both. Short has the lowest generalization, while Both is most likely in between Baseline and Long.

\section{EXPERIMENTS}

\section{A. Experiment 1}

The first experiment investigated the effect of different window sizes over which the MFCCs are calculated. This experiment gives insights into the effect of improving the temporal resolution in the MFCCs. In total four different MFCC representations were compared:

- Baseline: window size: $25 \mathrm{~ms}$; window shift: $10 \mathrm{~ms}$.

- Short: window size: $5 \mathrm{~ms}$; window shift: $2.5 \mathrm{~ms}$.

- Long: window size: $25 \mathrm{~ms}$; window shift: $2.5 \mathrm{~ms}$.

- Both: the Short and Long MFCCs are concatenated.

For all acoustic features, the input speech is first divided into overlapping Hamming windows of $25 \mathrm{~ms}$ or $5 \mathrm{~ms}$ with a $10 \mathrm{~ms}$ or $2.5 \mathrm{~ms}$ shift and a pre-emphasis factor of 0.97 . For the $25 \mathrm{~ms}$ windows $13 \mathrm{MFCCs}(\mathrm{C} 0-\mathrm{C} 12)$, and their first and second order derivatives were calculated (39 features). For the $5 \mathrm{~ms}$ windows, $7 \mathrm{MFCCs}(\mathrm{C} 0-\mathrm{C} 6)$ and first and second order derivatives were calculated (21 features). Afterwards, cepstral mean subtraction (CMS) was applied.

Adding context information has shown to improve classification performance (e.g., [13]). We therefore carried out different tests to determine the optimal amount of temporal context, which was $30 \mathrm{~ms}$ at both sides. For Baseline, 3 frames $(30$ $\mathrm{ms})$ left and right of a frame were taken into account resulting in MFCC vectors of length $7 * 39=273$. Since the window shift is different for the baseline system and the three other systems, the context was incorporated slightly differently in the Short, Long and Both classifiers: for these three classifiers \pm 3 frames were taken but taking only every fourth frame, in order to cover the same temporal context left and right of the frame as Baseline. This resulted in feature vectors of length 273 for Long and 147 for Short. To combine the window lengths ( $25 \mathrm{~ms}$ and $5 \mathrm{~ms}$ ) for Both, feature vectors of both windows with the same midpoint were concatenated, resulting in feature vectors of length 273 (from the $25 \mathrm{~ms}$ window) +147 (from the $5 \mathrm{~ms}$ window) $=420$.

\section{1) Results}

Table 4 shows the performance of the four AF classifiers in terms of percentage correctly classified frames on the TIMIT test material in confusion matrices. For clarity, the percentages along the diagonal are in bold. Comparing the three new acoustic features with the baseline system shows that the Short and Both classifiers performs best for 'burst' (Bur) as is to be expected, because the burst of a plosive is an event with a short duration. The Both and Long classifiers perform best for 'frication' (Fric). The AF value that profits most from adding temporal information is 'liquid' (Liq). Taking the different cell frequencies into account and computing the average accuracy, the Both classifier is slightly better than the others ( $85.1 \pm 0.1$ vs. BL: $84.0 \pm 0.2$, Short: $81.8 \pm 0.1$ and Long: $84.8 \pm 0.1 \%$ ). Most importantly, the Both classifier seems to be able to combine the classification power of both the Short and Long classifiers.
TABLE 4

CONFUSION MATRICES FOR THE AF CLASSIFIERS ON TIMIT; BL=THE BASELINE SYSTEM. THE DIAGONALS ALSO CONTAIN 95\% CONFIDENCE INTERVALS.

\begin{tabular}{|c|c|c|c|c|c|c|c|}
\hline BL & Sil & Liq & Gli & Nas & Fric & Bur & Clo \\
\hline Sil & $82.3 \pm .9$ & 0.2 & 1.4 & 0.3 & 2.4 & 3.3 & 10.1 \\
\hline Liq & 0.0 & $85.7 \pm .4$ & 4.1 & 3.9 & 3.0 & 2.1 & 1.2 \\
\hline Gli & 0.0 & 13.2 & $81.0 \pm .7$ & 2.9 & 0.9 & 1.0 & 1.0 \\
\hline Nas & 1.0 & 3.1 & 2.2 & $84.9 \pm .4$ & 3.0 & 0.9 & 4.9 \\
\hline Fric & 1.0 & 1.7 & 1.1 & 2.0 & $86.7 \pm .2$ & 4.8 & 2.7 \\
\hline Bur & 2.9 & 2.0 & 1.1 & 0.9 & 11.6 & $76.8 \pm .5$ & 4.7 \\
\hline Clo & 4.9 & 0.9 & 0.3 & 3.0 & 3.0 & 4.0 & $82.9 \pm .3$ \\
\hline Short & Sil & Liq & Gli & Nas & Fric & Bur & Clo \\
\hline Sil & $72.2 \pm .5$ & 0.1 & 1.9 & 0.9 & 5.1 & 6.0 & 13.8 \\
\hline Liq & 0.2 & $84.4 \pm .2$ & 5.2 & 4.8 & 3.1 & 1.2 & 1.1 \\
\hline Gli & 0.1 & 16.4 & $75.3 \pm .4$ & 4.0 & 1.9 & 1.1 & 1.2 \\
\hline Nas & 1.1 & 7.3 & 3.1 & $76.2 \pm .2$ & 5.1 & 0.9 & 6.3 \\
\hline Fric & 1.1 & 1.4 & 1.0 & 1.6 & $88.1 \pm .1$ & 4.8 & 2.0 \\
\hline Bur & 2.0 & 1.8 & 1.1 & 1.0 & 11.8 & $78.1 \pm .3$ & 4.2 \\
\hline Clo & 9.8 & 1.0 & 0.1 & 2.1 & 4.2 & 3.9 & $78.9 \pm .2$ \\
\hline Long & Sil & Liq & Gli & Nas & Fric & Bur & Clo \\
\hline Sil & $77.8 \pm .5$ & 0.0 & 1.4 & 0.0 & 3.1 & 4.8 & 12.9 \\
\hline Liq & 0.0 & $88.7 \pm .2$ & 3.9 & 3.6 & 1.8 & 1.1 & 0.9 \\
\hline Gli & 0.9 & 12.1 & $81.3 \pm .3$ & 2.8 & 0.9 & 1.0 & 1.0 \\
\hline Nas & 1.1 & 2.8 & 1.0 & $86.9 \pm .2$ & 3.2 & 0.1 & 4.9 \\
\hline Fric & 1.7 & 0.9 & 1.1 & 1.0 & $88.7 \pm .1$ & 4.0 & 2.6 \\
\hline Bur & 2.8 & 1.9 & 1.0 & 1.0 & 11.9 & $76.7 \pm .3$ & 4.7 \\
\hline Clo & 5.9 & 1.2 & 0.4 & 3.0 & 4.1 & 4.0 & $81.4 \pm .2$ \\
\hline Both & Sil & Liq & Gli & Nas & Fric & Bur & Clo \\
\hline Sil & $76.4 \pm .5$ & 0.2 & 1.0 & 0.0 & 2.9 & 4.8 & 14.7 \\
\hline Liq & 0.2 & $88.7 \pm .2$ & 4.1 & 3.2 & 1.8 & 1.1 & 0.9 \\
\hline Gli & 0.3 & 12.3 & $81.4 \pm .3$ & 2.8 & 1.0 & 1.0 & 1.2 \\
\hline Nas & 1.0 & 3.3 & 1.0 & $86.4 \pm .2$ & 3.1 & 0.2 & 5.0 \\
\hline Fric & 1.9 & 1.0 & 0.9 & 1.1 & $89.0 \pm .1$ & 2.7 & 3.4 \\
\hline Bur & 2.8 & 1.7 & 1.1 & 1.1 & 11.2 & $78.1 \pm .3$ & 4.0 \\
\hline Clo & 5.4 & 1.0 & 0.2 & 2.9 & 4.1 & 4.2 & $82.2 \pm .2$ \\
\hline
\end{tabular}

\section{2) Analysis}

As explained above, the synchronously changing AF values at the phone boundaries in the training data are likely to incur errors around those phone boundaries in the test material. In order to further analyze these errors and to investigate the influence of improving the temporal resolution of the MFCCs on these errors, the classification output of the best performing new classifier Both was further analyzed and compared to the Baseline system. To that end, the percentage correctly classified frames for the $20 \mathrm{~ms}$ following a phone boundary or leading towards a phone boundary are calculated for each of the AF values separately. As Baseline consists of a $10 \mathrm{~ms}$ 


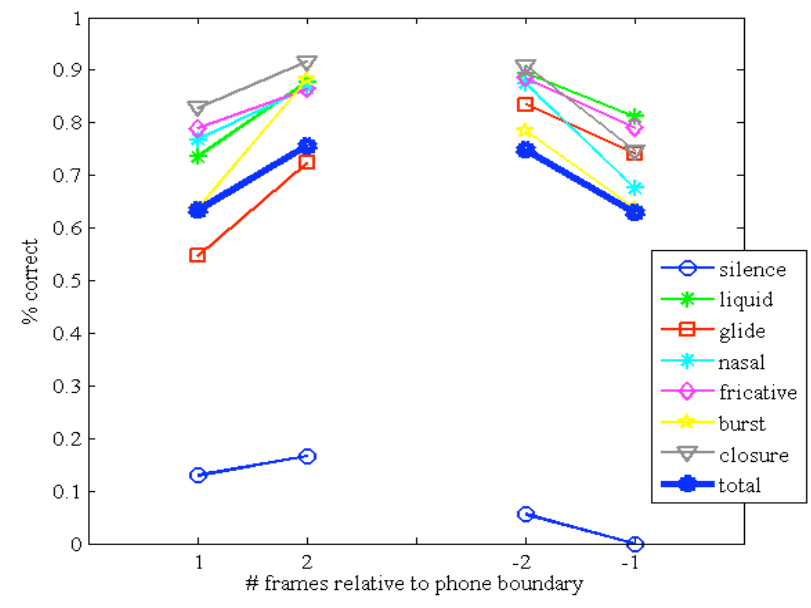

Fig. 1. The frame accuracy over time for each of the AF values for Baseline. The bold line shows the overall performance.

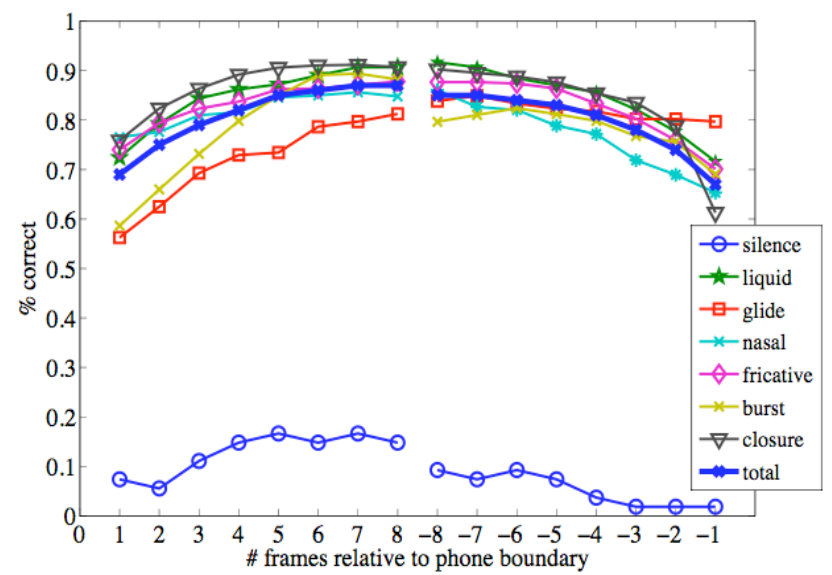

Fig. 2. The frame accuracy over time for each of the AF values for Both. The solid line shows the overall performance.

window shift, only two frames are analyzed; the Both system uses $2.5 \mathrm{~ms}$ window shifts, resulting in eight frames that are analyzed. With this method, only phonemes of a minimum duration of $40 \mathrm{~ms}$ could be analyzed, and the presented figures therefore only represent those $75.4 \%$ of segments in TIMIT that have this minimum length.

Figs. 1 (Baseline) and 2 (Both) show the percentage correctly classified frames for each of the AF values separately and the overall classification score over time. The positive numbers indicate the frame numbers counting from the start of the phone, the negative numbers indicate the frames numbers counted from the end of the phone. As expected, the performance of the classifiers is lower towards the boundaries as there the co-articulation effects are strongest. These results suggest that indeed the synchronously changing AFs in the test material result in a reduced frame accuracy. However, the central frames of the phone do not reach $100 \%$ correct classification accuracy; this might be due to the fact that the models are corrupted due to the synchronously changing AFs in the training material. While the frame accuracies over time for most AF values show the same pattern, there are a few exceptions. Silence shows extremely low accuracies close to the phone boundaries. The glides and bursts show lower accuracies at the beginning of the phone. Note that those AFs that do not follow the overall pattern are those that profit the least from the Both system compared to Baseline.

Finally, comparing Baseline and Both it can be observed that the first frame after a boundary and the last before a boundary have relatively low accuracies and that the accuracies for some manner classes rise at a different rate than others. However, all in all, the results seem to suggest that increasing the temporal resolution yields a larger proportion of representative training data which results in better classifiers which are less sensitive to errors introduced by the synchronously changing AFs.

\section{B. Experiment 2}

In the second experiment, the best performing new acoustic features are compared with the baseline system on the SVArticulatory data. To that end, new SVMs are trained using the acoustic features of the Both system and the Baseline system on the SVArticulatory data and subsequently tested in a 5-fold cross validation scheme. Folds were generated by randomly dividing the whole dataset into five parts. Note that, as opposed to the TIMIT experiment, there was not a stringent separation between utterances from training and test speakers. Both classifiers were trained five times on $80 \%$ of the material and tested on the remaining $20 \%$. Each time, $\gamma$ and $c$ parameters were optimized on the training material by training and testing on two random subsets (from the training set) with different parameters.

With this experiment we intend to investigate the impact of labeling accuracy and its interaction with improved temporal resolution.

\section{3) Results and Discussion}

Table 5 shows the performance of Baseline and Both in terms of percentage correctly classified frames on the SVArticulatory material in confusion matrices. As is clear from the percentages on the diagonal, Both outperforms Baseline for all AF values, except for silence (Sil) where they perform equally well. The performance of Both on the SVArticulatory data is substantially better than on TIMIT. Despite the differences between the two experiments we firmly believe that this is mainly due to more accurate labels in SVArticulatory than in TIMIT. Furthermore, the difference between Both and Baseline is much bigger than for the TIMIT data. The difference is biggest for 'burst' (Bur), which is according to expectation as here the temporal resolution resulting from the short windows gives additional information about the bursts, which are short and dynamic acoustic events. Thus, it seems safe to conclude that when the training material does allow for asynchronously changing (and consequently more accurate) AFs (SVArticulatory), the upper bound for the classification performance is raised. Moreover, in the presence of more accurate labels in training and test the gain obtained thanks to improved temporal resolution is larger than the improvement obtained in TIMIT, where the AF labels are less accurate because of the synchronous changes imposed by the mapping from phonetic symbols to AF labels. 
TABLE 5

CONFUSION MATRICES FOR THE TWO AF CLASSIFIERS ON SVARTICULATORY; $\mathrm{BL}=$ THE BASELINE SYSTEM.

\begin{tabular}{cccccccc}
\hline BL & Sil & Liq & Gli & Nas & Fric & Bur & Clo \\
\hline Sil & $\mathbf{9 6 . 2 \pm . 4}$ & 0.2 & 0.0 & 1.0 & 1.3 & 1.1 & 0.2 \\
Liq & 1.0 & $\mathbf{8 9 . 9} \pm \mathbf{2 . 3}$ & 3.7 & 1.8 & 1.7 & 1.0 & 0.9 \\
Gli & 1.9 & 2.5 & $\mathbf{8 7 . 0 \pm . 2 . 7}$ & 3.6 & 2.1 & 1.9 & 1.0 \\
Nas & 1.0 & 1.9 & 2.9 & $\mathbf{8 4 . 4 \pm 2 . 5}$ & 3.7 & 1.1 & 5.1 \\
Fric & 1.9 & 1.4 & 1.8 & 1.9 & $\mathbf{8 3 . 1} \pm .1 .8$ & 4.8 & 5.1 \\
Bur & 1.1 & 1.0 & 0.2 & 1.4 & 3.5 & $\mathbf{8 2 . 7 \pm 3 . 6}$ & 10.1 \\
Clo & 1.8 & 0.8 & 1.9 & 3.2 & 5.9 & 2.9 & $\mathbf{8 3 . 5} \pm \mathbf{2 . 1}$ \\
\hline
\end{tabular}

\begin{tabular}{cccccccc}
\hline Both & Sil & Liq & Gli & Nas & Fric & Bur & Clo \\
\hline Sil & $\mathbf{9 6 . 0 \pm . 2}$ & 0.4 & 0.1 & 0.1 & 2.3 & 0.1 & 1.0 \\
Liq & 1.0 & $\mathbf{9 5 . 4 \pm . 8}$ & 1.2 & 0.9 & 0.9 & 0.4 & 0.2 \\
Gli & 0.2 & 1.9 & $\mathbf{9 5 . 1 \pm . 9}$ & 1.4 & 1.0 & 0.2 & 0.2 \\
Nas & 0.2 & 1.9 & 0.9 & $\mathbf{9 3 . 3 \pm . 9}$ & 1.5 & 0.2 & 2.0 \\
Fric & 1.9 & 1.1 & 0.0 & 0.9 & $\mathbf{8 9 . 3} \pm .7$ & 3.3 & 3.5 \\
Bur & 0.2 & 0.1 & 0.0 & 0.0 & 4.2 & $\mathbf{9 2 . 4 \pm 1 . 3}$ & 3.1 \\
Clo & 1.4 & 0.2 & 0.9 & 1.2 & 5.1 & 1.2 & $\mathbf{9 0 . 0 \pm . 9}$ \\
\hline
\end{tabular}

\section{CONCLUSIONS}

The aim of the present study was two-fold. The first aim was to build an AF classifier that can be used for reliable and accurate detection of slight pronunciation errors and the automatic analysis of fine-phonetic detail. In order to improve the automatic classification of the manner of articulation, we proposed a combination of acoustic features with both a high temporal and a high frequency resolution so that it becomes possible to detect and reliably classify articulatory events of short duration, such as bursts in plosives. The results showed that combining MFCCs derived from a long window of $25 \mathrm{~ms}$ and from a short window of $5 \mathrm{~ms}$ that are both shifted with $2.5 \mathrm{~ms}$ steps outperforms standard MFCCs. The added value of temporal information was found when testing the SVM classifiers on TIMIT and on a subset of SVArticulatory.

Secondly, we investigated the effect of the AF labeling of the training and test material on performance estimates. Comparing the results of the two presented experiments showed that for classifiers trained on data that is labeled at the AF level with asynchronously changing labels (SVArticulatory) the improvement from Baseline to Both is larger than for classifiers trained on data where the labels of the AF level were generated by mapping from the phone label to the AF value (TIMIT). Thus in order to train reliable and accurate $\mathrm{AF}$ classifiers, training and test material that allows for asynchronously changing AFs is crucial.

\section{ACKNOWLEDGMENTS}

Barbara Schuppler is supported by the Marie Curie Research Training Network Sound-to-Sense. Joost van Doremalen is supported by the project DISCO, funded by the Dutch-Flemish programme STEVIN. Odette Scharenborg is supported by a Veni-grant from the Netherlands Organization for Scientific Research (NWO).

\section{REFERENCES}

[1] M. Ostendorf, "Moving beyond the 'beads-on-a-string' model of speech," IEEE ASRU Workshop, 1999, pp. 79-84.

[2] M. Saraçlar, H. Nock, S. Khudanpur, "Pronunciation modelling by sharing gaussian densities across phonetic models," Computer Speech and Language, 14, pp. 137-160, 2000.

[3] K. Kirchhoff, "Robust speech recognition using articulatory information," Ph.D. thesis, University of Bielefield, Germany, 1999.

[4] M. Wester, J. Frankel, S. King, "Asynchronous articulatory feature recognition using dynamic Bayesian networks," Proc. IEICI Beyond HMM Workshop, 2004

[5] K. Kirchhoff, G. A. Fink, G. Sagerer, "Combining acoustic and articulatory feature information for robust speech recognition," Speech Communication, 37, pp. 303-319, 2002.

[6] K. Kirchhoff, "Combining articulatory and acoustic information for speech recognition in noisy and reveberant environments," Proc. ICSLP, 1998, pp. 891-894.

[7] S. M. Siniscalchi, T. Svendsen, C.-H. Lee, "Towards a detector-based universal phone recognizer," Proc. ICASSP, 2008, pp. 4261-4264.

[8] O. Scharenborg, "Using durational cues in a computational model of

[9] spoken-word recognition," Proc. Interspeech, 2009.

[10] J. Tepperman, S. Narayanan, "Using Articulatory Representations to Detect Segmental Errors in Nonnative Pronunciation," IEEE Trans. on Audio, Speech, and Language processing, 16 (1), pp. 8-22, 2008.

[11] B. Schuppler, W. van Dommelen, J. Koreman, M. Ernestus, "Wordfinal [t]-deletion: An analysis on the segmental and sub-segmental level," Proc. Interspeech, 2009.

[12] K. Truong, A. Neri, C. Cucchiarini, H. Strik, "Automatic pronunciation error detection: an acoustic-phonetic approach," Proc. InSTIL/ICALL, Venice, Italy, 2004, pp. 135-138.

[13] O. Scharenborg, V. Wan, R. K. Moore, "Towards capturing fine phonetic variation in speech using articulatory features," Speech Communication, 49, 2007, 811-826.

[14] J. Frankel, M. Wester, S. King, "Articulatory feature recognition using dynamic bayesian networks," Computer Speech and Language, 21 (4), pp. 620-640, 2007.

[15] O. Scharenborg, M. P. Cooke, "Comparing human and machine recognition performance on a VCV corpus," Proc. Workshop on Speech Analysis and Processing for Knowledge Discovery, 2008.

[16] F. Pernkopf, T. V. Pham, J. A. Bilmes, "Broad phonetic classification using discriminative bayesian networks," Speech Communication, 51, pp. 151-166, 2009.

[17] T. Pruthi, C. Y. Espy-Wilson, "Acoustic parameters for automatic detection of nasal manner," Speech Communication, 43, pp. 225-239, 2004.

[18] J. Garofolo, "Getting started with the DARPA TIMIT CD-ROM: An acoustic phonetic continuous speech database," National Institute of Standards and Technology (NIS), Gaithersburgh, MD, 1988.

[19] S. King, C. Bartels, J. Bilmes, "SVitchboard 1: Small vocabulary tasks from switchboard 1," Proc. Interspeech, 2005, pp. 3385-3388.

[20] K. Livescu et al., "Manual transcriptions of conversational speech at the articulatory feature level," Proc. ICASSP, 2007.

[21] S. King, C. Bartels, J. Bilmes, "SVitchboard 1: Small vocabulary tasks from switchboard 1," Proc. Interspeech, 2005, pp. 3385-3388.

[22] S. King, P. Taylor, "Detection of phonological features in continuous speech using neural networks," Computer Speech and Language, 14, pp. 333-353, 2000.

[23] S. M. Siniscalchi, T. Svendsen, C.-H. Lee, "Towards bottom-up continuous phone recognition," Proc. ASRU, 2007 pp. 566-569.

[24] J. Frankel, M. Magimai-Doss, S. King, K. Livescu, Ö. Çetin, "Articulatory feature classifieres trained on 2000 hours of telephone speech," Proc. Interspeech, 2007, pp. 2485-2488.

[25] C. Burges, "A Tutorial on Support Vector Machines for Pattern Recognition," Data Mining and Knowledge Discovery, 2 (2), 1-47, 1998.

[26] C.-C. Chang, C.-J. Lin, LIBSVM: a library for support vector machines, 2001. Software available at http://www.csie.ntu.edu.tw/ cjlin/libsvm.

[27] L. Deng, D. Yu and A. Acero, "Structured Speech Modeling", IEEE Transactions on Audio, Speech and Language Processing, 14 (5), 2006. 